\title{
Extrahepatic manifestations of hepatitis C virus (HCV)
}

\author{
David G Samuel, Ian W Rees
}

Department of Gastroenterology, Prince Phillip Hospital, Llanelli, UK

\section{Correspondence to}

Dr D G Samuel, Department of Gastroenterology, Prince Phillip Hospital, Llanelli Sa14 8QF, UK; daisams@doctors.org.uk

Received 4 February 2013 Revised 21 April 2013 Accepted 25 April 2013 Published Online First 24 May 2013
To cite: Samuel DG, Rees IW. Frontline Gastroenterology 2013;4:249-254.

\begin{abstract}
Hepatitis $C$ virus (HCV) is an infectious disease that often remains asymptotic and unrecognised until complications of the virus arise. These often include extrahepatic manifestations of the virus, which first bring patients into contact with the medical profession. First recognised in the 1990s several syndromes and conditions have now been linked to hepatitis $C$, while others are still emerging. In some patients, extrahepatic manifestations can be the dominant feature, while hepatic disease is mild. Some conditions have an established association with the virus with a proven pathophysiological and epidemiology, such as cryoglobulinaemia. Others have consistently been found to be seen in patients with $\mathrm{HCV}$, but the underlying cause of these conditions is not clearly understood. These include porphyria cutanea tarda. Many other autoimmune conditions are commonly seen in the patients with HCV as well as nephropathies, but the exact interplay between virus and resulting clinical condition is not clear. Clinicians have to have a high index of suspicion and a knowledge of the extrahepatic manifestations of $\mathrm{HCV}$ in order to not only treat the manifestation but also in initiated timely therapies for the underlying $\mathrm{HCV}$.
\end{abstract}

\section{INTRODUCTION}

Hepatitis $\mathrm{C}$ virus (HCV) is an infectious disease that often remains asymptotic and unrecognised until complications of the virus arise. These often include extrahepatic manifestations (EHM) of the virus, which first bring patients into contact with the medical profession. ${ }^{1}$ Up to $75 \%$ of patients will develop an EHM during their illness. First recognised in the 1990s several syndromes and conditions have now been linked to hepatitis C, while others are still emerging. In some patients, EHM can be the dominant feature, while hepatic disease is mild. ${ }^{2}$
Some conditions have an established association with the virus with a proven pathophysiological and epidemiology such as cryoglobulinaemia.

Others have consistently been found to be seen in patients with $\mathrm{HCV}$, but the underlying cause of these conditions is not clearly understood. These include porphyria cutanea tarda. Many other autoimmune conditions are commonly seen in patients with HCV as well as nephropathies, but the exact interplay between virus and resulting clinical condition is not clear. Among these, glomerulonephritis is an important condition to consider (table 1).

This review aims to provide an overview of the conditions with the greatest level of evidence supporting a direct link with HCV, as well as looking at the potentially most life-threatening manifestations, with a focus on investigations and management. I have selected conditions, which have the greatest amount of pathophysiological evidence for a causal effect by the HCV, as looking at multiple conditions would be beyond the scope of a single paper, but the principles of investigations and management apply to many of the other conditions listed above. The paper provides a helpful overview for the hepatology clinic as well as encounters on general medical takes.

Before looking at the individual conditions focused on in this article, I will provide a brief overview of the current evidence suggesting the pathophysiology of extrahepatic tissue invasion and pathogenesis of the HCV.

\section{THE PATHOPHYSIOLOGY OF EHM OF HCV}

It is thought that extrahepatic tissues serve as a reservoir for the hepatitis virus, but especially tropism for the lymphoid 
Table 1 The extrahepatic conditions related to hepatitis $C^{12}$

\begin{tabular}{lllll}
\hline Haematological & Systemic & Rheumatology & Dermatology & Organ dysfunction \\
\hline Cryoglulinaemia & Arthralgia & Bechet's disease & Canities & Diabetes \\
Membranoproliferative glomerulonephritis & Arthritis & Vasculitis & Lichen myxoedematosus & Cardiomyopathy \\
Multiple myeloma & Fatigue & Raynaud's syndrome & Porphyria cutanea tarda & Idiopathic lung fibrosis \\
Neutropenia/thrombocytopenia & Fibromyalgia & Sialadenitis & Pruritis & Peripheral neuropathy \\
Non-Hodgkin's lymophoma & Corneal ulceration & Systemic lupus erythematous & Spider naevi & Hypothyroidism \\
Waldenstrom Macroglobulinaemia & & Vitiligo & Renal failure & \\
\hline
\end{tabular}

tissues. ${ }^{2}$ Like other viruses, the HCV has developed an ability to evade detection by the immune system, which in turn leads to chronic inflammation, tissue damage and immune-complex aggregation, activating autoimmune phenomena. ${ }^{2} 3$ In cryoglubulinaemia, which will be discussed in detail below, B-cell lymphoproliferation is the initial immune-cascade trigger. The HCV tropism allows the lymphocytes to serve as host and reservoir, with the virus thought to bind to the CD81 ligand of the B lymphocyte using an E2 protein. ${ }^{4}$ Flint was the first to show that this engagement with the HCV envelope activates lymphocytes and subsequently leads to immunoglobulin production.

\section{CRYOGLOBULINAEMIA}

Cryoglobulinaemia is among the most common and closely linked extrahepatic HCV manifestations and is most often a mixed cryoglobulinaemia of type 2 or $3 .^{2} 5$ Considered a lymphoproliferative disorder, it is thought to result from chronic stimulation and over production of $\mathrm{B}$ cells ${ }^{6}$ producing immunoglobulin. The disorder is characterised by these abnormal bloodborne protein, which precipitate and aggregate into gel-like substances at reduced temperatures, normally aggregate below $37^{\circ} \mathrm{C} .^{2}{ }^{4}$ When the cryoglobulins aggregate and form gel-like substances cause clinical signs and complications through occluding vessels and inflicting target organ damage. ${ }^{7}$ Symptoms relate to the organ affected and can range from joint pain, muscle pain, acute kidney injury and

\section{Curriculum outcomes}

\section{Viral hepatitis}

To be aware of hepatitis C and B, those individuals at risk and the principles of treatment

- understands the serological interpretation, categorisation and investigation of patients with chronic hepatitis $C$ with particular emphasis on the need for treatment and surveillance

- uses appropriate diagnostic modalities including serology, genotyping, viral load measurements, liver biopsy and related investigations. glomerulonephritis, or more commonly manifesting as a vasculitic syndrome. Critical ischaemia can result with loss of limbs, particularly digits. ${ }^{17}$ Other nonspecific symptoms including lethargy are common, with the most common symptoms including arthralgia and pruritus in up to $18 \%$ of patients. Up to $50 \%$ of patients remain relatively asymptomatic until a critical assault on an organ occurs ${ }^{16}$ (table 2).

At first presentation of cryoglobulinaemia investigating for the cause would include a hepatitis screen. Other investigations include urinalysis, which sometimes shows haematuria, a complement assay, which is low, cryoglobulin levels if available within local hospitals and a full blood count. Rheumatoid factor is often positive in types 2 and 3 and can sometimes point to coexisting disease ${ }^{4}$ (table 3 ).

Biopsies typically show a leukoclastic vasculitis, which usually involves the medium-sized vessels. ${ }^{7} 8$

Another important manifestation of cryoglobulinaemia is a peripheral neuropathy, which has been reported in up to $90 \%$ of patents depending on the case series. ${ }^{9}$ Most cases involve a sensory impairment similar to the diabetic neuropathy, and also in a similar glove stocking distribution, but not exclusively. ${ }^{9}$ The condition can be made worse by interferon therapy, which is an important issue to consider in managing the underling $\mathrm{HCV}^{10}$ A biopsy typically shows axonal damage and an epineural vasculitic infiltrate. $^{7} 10$

Therapy is targeted at

A. treating the underlying $\mathrm{HCV}$ and reducing or eradicating viral load

B. immune-targeted therapy.

The most widely studied and used regimes include cyclophosphamide along with steroid therapy with the aim of suppressing antibody production and cryoglobulin production, but with mixed results. ${ }^{2}{ }^{8} 11$ A major concern and drawback of this therapy is the

Table 2 The immunological profile and classification of cryoglobulinaemia ${ }^{6}$

\begin{tabular}{ll}
\hline Cryoglobulinaemia class & Clonal precipitates \\
\hline $\begin{array}{l}\text { Type I (lymphoproliferative) } \\
\text { Type II—-mixed }\end{array}$ & $\begin{array}{l}\text { Isolated monoclonal IgG or IgM } \\
\text { Polyclonal immunoglobulins (IgG } \\
\text { and monoclonal IgA, IgM, IgG } \\
\text { Polyclonal IgM and IgG }\end{array}$ \\
\hline
\end{tabular}


Table 3 The initial investigations to consider in a patient presenting with the symptoms and clinical signs of cryoglobulinaemia $^{4}$

\begin{tabular}{ll}
\hline Cryoglobulin levels & HCV antibody \pm PCR and virology \\
\hline Immunoglobulin and protein levels & Urine analysis \\
Complement levels & Biopsy of lesion (within $48 \mathrm{~h}$ ) \\
Gammopathy assessment & Biochemistry \\
\hline
\end{tabular}

Adapted from Khattab et al. ${ }^{4}$

$\mathrm{HCV}$, hepatitis C virus.

risk of allowing HCV viral replication and the most recent review of therapy does not endorse cyclophosphamide therapy. ${ }^{12}$ More targeted therapies such as rituximab have shown greater promise and has been shown to rapidly reduce antibody and cryoglobulin levels. ${ }^{13}{ }^{14}$ Severe cases, or those involving major organs, warrant early corticosteroid therapy and immunosuppression therapy. ${ }^{12}$ Patients are often treated with plasmapheresis ${ }^{14}$ (see next section). Treating the underlying HCV remains the cornerstone of preventing both hepatic and extrahepatic complications (figure 1).

\section{GLOMERULONEPHRITIS}

Glomerulonephritis is defined as an inflammatory process which, in the case of hepatitis $\mathrm{C}$, is due to immune dysregulation and an ineffective response by the body to deal with the HCV. ${ }^{5}{ }^{15}$ The most common glomerulonephritis associated with HCV-related cryoglobulinaemia is membranoproliferative glomerulonephritis. ${ }^{11}$ The pathophysiology of HCV-related glomerulonephritis is believed to involve deposition of immune complexes, anti-HCV immunoglobulin and an IgM subtype rheumatoid factor. ${ }^{13}$

Presentation ranges from hypertension, which is present in $80 \%$ of patients with an associated moderate renal insufficiency. ${ }^{13} 20 \%-35 \%$ of patients may present as a nephrotic syndrome and up to a quarter of patients will be nephritic. ${ }^{4}{ }^{15}$ However, $10 \%$ of patients will present with rapidly deteriorating renal

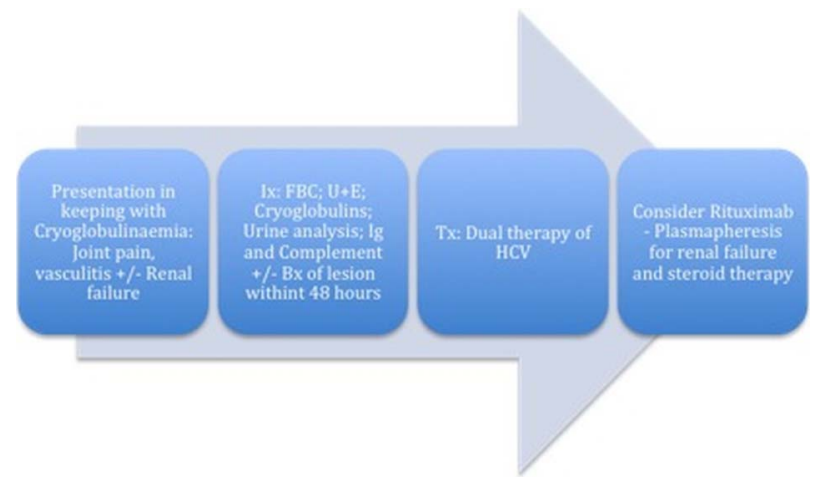

Figure 1 An algorithm for investigating and managing patients with cryoglobulinaemia. ${ }^{2} 24101314$ function and warrant urgent referral to specialist nephrology services (table 4).

Treatment is underpinned by aggressive hypertensive therapy with associated renin-angiotensin system blockade. ${ }^{15}$ This is achieved using ACE inhibitors, angiotensin receptor blockers and diuretics. Patients tend to have high lipid and triglyceride levels and managing coronary risk factors have been proven to be beneficial. ${ }^{15} 16$ Treating the underlying HCV infection has not only been shown to clear the virus successfully but also in preventing or limiting renal damage. ${ }^{15}$ Dual therapy with interferon and ribavirin has been shown to reduce viral load, as well as reduce proteinuria but has mixed success in improving glomerular filtration and creatinine levels, but studies have consistently shown that commencing antiviral therapy stabilises renal function. ${ }^{17}$ Data also support prolonged therapy for at least 48-52 weeks, irrespective of early reductions in HCV loads at 12 weeks, but therapy can be considered for as long as 72 weeks in patients with non-responding viral load, but with biochemical and clinical improvements. ${ }^{14}$ Caution has to be taken in using ribavirin at full dose as clearance is related to renal function. ${ }^{14} 17$

In patients who present with rapidly progressive glomerulonephritis and nephritic syndrome, early aggressive therapy is crucial. ${ }^{12}$ Plasma exchange is used in order to remove circulating cryoglobulins and hence reducing the insult on organs and tissues. ${ }^{13}$ This is normally carried out by exchanging 3 litres, three times a week over 2-3 weeks. Pulsed steroid therapy in conjunction with plasma exchange has also been shown to be beneficial in correcting glomerular filtration dysfunction. ${ }^{7}$ Cyclophosphamide has been used to suppress B cell function during the acute phase of the illness by reducing cryoglobulin production. ${ }^{10} 18$ However, as discussed previously, more recent studies have not provided evidence to support its recommendation and is not currently recommended as first-line therapy ${ }^{14}$ More recent studies have looked at the potential role of rituximab in treating nephritic syndrome and glomerulonephritis. The rationale behind this research is the impact that the CD20 targeted antibody has on selectively targeting $\mathrm{B}$ cells, the driving force in cryoglobulinaemias. ${ }^{18}$ Small studies have shown mixed benefits in reducing proteinuria and stabilising renal function, but no large randomised control studies have yet shown clear benefit and concerns remain over the potential to predispose patients to overwhelming sepsis. ${ }^{13} 1718$ There also remains concern that suppressing immune function leads, while improving renal disease, paradoxically increases circulating viral load, which has been shown in cyclophosphamide, ${ }^{14}$ but not yet shown in rituximab.

Unlike the HCV, prognosis with glomerulonephritis is relatively poor and up to $50 \%$ of patients will progress to end stage renal disease ${ }^{15}$ (figure 2). 
Table 4 The typical findings and differential features of mesangiocapillary and rapidly progressive glomerulonephritis (RPGN) ${ }^{15}$

\begin{tabular}{lllllll}
\hline Glomerulonephritis & Proteinuria & Nephrotic & Nephritic & Haematuria & ARF & CRF \\
\hline Mesangiocapillary & ++ & ++ & + & + & + & ++ \\
Rapidly Progressive & + & \pm & +++ & ++ & ++ & + \\
\hline
\end{tabular}

Very common $=+++$; quite common $=++$; common $=+$; rare or never seen $=-{ }^{15}$

$A R F$, acute renal failure; $C R F$, chronic renal failure.

\section{PORPHYRIA CUTANEA TARDA}

Porphyria cutanea tarda (commonly referred to as PCT) is recognised as the most prevalent subtype of porphyritic diseases. The disease is characterised by onycholysis and blistering of the skin in areas that receive higher levels of exposure to sunlight. ${ }^{19}{ }^{20}$ The primary cause of this disorder is a deficiency of uroporphyrinogen decarboxylase (UROD), a cytosolic enzyme that is a step in the enzymatic pathway that leads to the synthesis of haem. ${ }^{19}$ The disorder results from low levels of the enzyme responsible for the fifth step in haem production. ${ }^{20}$ Haem is a vital molecule for all of the body's organs. It is a component of haemoglobin, the molecule that carries oxygen in the blood. Excess circulating iron has been shown to enhance toxic metabolite formation, which include oxidation products which inhibit UROD. ${ }^{19}$

Typically, patients who are ultimately diagnosed with PCT first seek treatment following the development photosensitivities in the form of blisters and erosions on commonly exposed areas of the skin. ${ }^{19} 21$ This is usually observed in the face, hands, forearms and lower legs. It heals slowly and with scarring. ${ }^{20}$ Though blisters are the most common skin manifestations of PCT, other skin manifestations like hyperpigmentation (as if they are getting a tan) and hypertrichosis (mainly on top of the cheeks) also occur. ${ }^{19}$ PCT is a chronic condition, with external symptoms often subsiding and recurring as a result of a number of factors. In addition to the symptomatic manifestation of the disease in the skin, chronic liver problems are extremely common in patients with the sporadic form of PCT. ${ }^{20}$

\section{DIAGNOSIS}

While the most common symptom of PCT is the appearance of skin lesions and blistering, their appearance in isolation cannot lead to a conclusive diagnosis. ${ }^{19} 22$ Laboratory testing will commonly reveal high

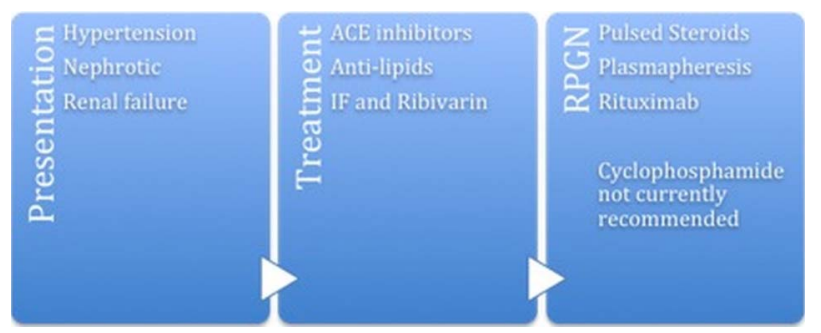

Figure 2 The treatment algorithm for patients presenting with glomerulonephritis. ${ }^{13151718}$ levels of uroporphyrinogen in the urine, clinically referred to as uroporphyrinogenuria. ${ }^{19} 21$

Treatment options include avoidance of sunlight, which precipitates the typical rash associated with PCT. In addition, smoking cessation and avoidance from alcohol is advised, especially in patients with hepatitis $\mathrm{C},{ }^{19}$ although evidence to strongly support these measures specifically to PCT is limited. ${ }^{23}$ More targeted treatments include regular venosection, with the aim of reducing iron stores to the lower limit of normal. ${ }^{20}$ This in turn improves haem synthesis disturbed by ferro-mediated inhibition of UROD. ${ }^{23}$ Clinicians must be careful not to cause anaemia in patients; therefore, interval phlebotomy must be tailored to the individual. Reducing the iron stores, and therefore, the potential detrimental effect on hepatocytes has been shown to improve the efficacy of HCV therapy. ${ }^{21}$ A unit of blood is normally removed between twice weekly and once 3 weekly intervals with regular blood counts taken to avoid inducing anaemia, particularly pertinent if being treated with ribavirin. ${ }^{20} 23$ This is also the preferred therapy of choice in patients with a significant iron load.

If phlebotomy is not convenient or is contraindicated or for patients with relatively mild iron overload, oral chloroquine phosphate (125-250 mg per oral (PO) twice weekly) or hydroxychloroquine sulfate (100-200 mg PO 2-3 times/week), doses much lower than those used for antimalarial or photoprotective indications, can be effective, although significant caution has to be applied in patients with hepatitis C, where hepatoxicity can occur even at low doses of these medications. ${ }^{22}{ }^{23}$ In obese patients, doses should be adjusted to ideal weight in order to avoid toxicity. Remission is often seen within 6-12 months. ${ }^{23}$ All patients should receive regular ocular examination both before and during therapy, as recommended by the Royal College of

Table 5 The monitoring protocol required for patients receiving hydroxychloroquinine therapy (taken from BNF 62 September 2012)

\begin{tabular}{ll}
\hline Before initiating therapy & During therapy monitoring \\
\hline Baseline renal and hepatic function & Review vision and note changes \\
Visual check and optometry review & Reading chart comparison \\
Visual acuity assessed using standard & Slit lamp examination if indicated \\
chart & to assess for uveitis \\
Initiate dose at max $6.5 \mathrm{mg} / \mathrm{kg}$ & Long-term therapy- \\
& ophthalmology assessment on a \\
& yearly basis \\
\hline
\end{tabular}




\section{Multiple choice questions}

1. A male patient presented to his general practitioner with painful joints, lethargy and cold digits. He had noticed that working outside in the cold weather over the recent weeks had made the symptoms much worse and that returning home at night did ease the problem slightly. More worryingly he had developed a generalised red rash and an ulcer on one of his fingers, which had turned black over $24 \mathrm{~h}$. A renal function test taken 6 months previously was normal but on presentation his urea was elevated at 10.9 and his creatinine was 189 .

The following tests should be performed in making the diagnosis:

i. HIV test, cryoglobulin levels and a hepatitis screen

ii. Renal biopsy, ultrasound of the kidneys and renal angiography

iii. Coombs test, clotting screen and serum electrophoresis

iv. Urine legionnaire antibody, chest $x$-ray and renal biopsy

v. Biopsy of the skin rash, Anti-neutrophil cytoplasmic antibody (ANCA) and a blood film

Answer: i) This patient presents with the signs and symptoms of cryoglobulinaemia. The most important underlying conditions to diagnose are hepatitis and HIV in order to initiate prompt treatment. Treatment should not include cyclophosphamide as current evidence does not show a benefit, and there is a risk of worsening $\mathrm{HC}$ viral load. Steroid therapy is commonly used, although rituximab is being used more and more. However, the drawback of these therapies is the potential to allow the HIV virus, and the hepatitis virus to rapidly develop. Therefore, diagnosis is crucial for appropriate treatment and monitoring. All therapies have been shown to rapidly reduce cryoglobulin levels and antibody production, but rituximab is more targeted therapy suppressing B-lymphocyte activity.

2. A female intravenous drug user had been diagnosed with HCV 6 months with a relatively low level or viraemia ( $<800$ copies). However, she presented to her hepatologist after requesting an urgent appointment feeling unwell with regular headaches. She was not currently on treatment for her hepatitis $\mathrm{C}$ and wondered if the virus was giving her headaches. She had also noticed her ankles swelling far more in the previous week and her urine had becoming frothy. In clinic, her urinalysis reveals protein ++++ and her BP is 210/105. You believe she has developed glomerulonephritis and initiate the following treatment:

i. ACE inhibitor therapy, cyclophosphamide and dual antiviral therapy

ii. Diuretic therapy and follow-up in 1 week time

iii. ACE inhibitor, diuretic therapy and an lumbar puncture

iv. Ace inhibitor therapy, cyclophosphamide, dual antiviral therapy and antilipid therapy

v. None of the above
Answer: iv) This patient has developed glomerulonephritis and required urgent treatment to prevent rapidly progressive disease and renal failure. Aggressive antihypertensive therapy underpins the early management. Although studies do not strongly support its' use, cyclophosphamide which suppresses B cell function and cryoglobulin production is still used in some centres but not recommended as best practice. Treating the underlying HCV with dual therapy (with ribavirin adjusted according to renal impairment) has not only been shown to clear the virus but reduce the incidence of progression to end-stage renal disease. As patients often have high TAG and cholesterol levels, managing coronary disease risk factors is crucial in a traditionally younger population.

3. A female patient, who had been diagnosed with HCV 18 months ago but had not yet started therapy due to ongoing depression and substance misuse, presented to the emergency department with arthralgia, lethargy and abdominal pain which had localised to the loins over $24 \mathrm{~h}$. On admission she was febrile, hypertensive with a BP of 220/98, had macroscopic haematuria and when catheterised produced frothy urine. Initial renal function showed a raised creatinine at 168 but a repeat sample $6 \mathrm{~h}$ after admission showed this had risen to 230 . A working diagnosis of glomerulonephritis was made. Definitive treatment would be likely to involve:

i. $\quad 5-10 \mathrm{mg}$ ramipril, ultrasound of the renal tract and if evidence of hydronephrosis - placement of a ureteric stent

ii. $\quad 2.5-10 \mathrm{mg}$ ramipril and prednisolone $40 \mathrm{mg}$

iii. Hemofiltration, cyclophosphamide and renal biopsy

iv. Ramipril, plasma exchange therapy and consideration for steroid therapy

v. Plasma exchange therapy, furosemide and fluid restriction

Answer: iv) This patient has developed glomerulonephritis, which appears to be rapidly progressive. She requires rapid intervention, preferably in a high-dependency setting and should be considered for urgent plasma exchange therapy along with consideration for pulsed steroid therapy. This combination has been shown to reduce glomerular dysfunction. Antihypertensive therapy should be initiated early irrespective of the degree of renal impairment.

4. A male patient returns from a holiday with his wife and presents to his GP with a red, blistering rash which has appeared up his tan lines and covering his face. He reports he normally 'tans well' and does not normally get sunburn. Further questioning about past medical problems is unremarkable, although he has spent large proportions of his career abroad in Indonesia, the Philippines and Thailand. You believe he may be suffering from a sporadic form of $\mathrm{PCT}$ and wonder if the underlying cause is hepatitis C. After consenting to send away a hepatitis screen you also send away the following tests to help confirm the diagnosis and aid management: 
i. Skin biopsy and serous fluid analysis from blisters

ii. Urine analysis, Full blood count (FBC) and Liver Function tests (LFTs)

iii. Ferritin levels, urine analysis and skin biopsy

iv. Urine analysis, $F B C$ and skin biopsy

v. Skin biopsy and ferritin levels.

Answer: ii) The diagnosis of PCT is often made on clinical presentation of typical photosensitive rash. A high level of urinary uroporphyrinogen can support the diagnosis. Treatments are tailored depending on severity and convenience for the patient, but both venosection (which causes anaemia) and topical or oral antimalarials (can cause hepatotoxicity) mean basic assessment with bloods need to be done to guide treatment options.

Ophthalmologists. The following steps should be taken when initiating and monitoring hydroxychloroquine therapy (table 5).

\section{SUMMARY AND CONCLUSIONS}

While hepatitis $C$ is typically associated with liver dysfunction and progression to cirrhosis, its clinical presentation remains diverse and sometimes unexpected. Clinicians have to have a high index of suspicion and a knowledge of the EHM of HCV in order to not only treat the manifestation, but in initiated timely therapies for the underlying $\mathrm{HCV}$.

Contributors DGS wrote the main body of the article and advice and review was provided by IWR.

\section{Competing interests None.}

Provenance and peer review Not commissioned; internally peer reviewed.

\section{REFERENCES}

1 Cacoub P, Renou C, Rosenthal E, et al. Extrahepatic manifestations associated with hepatitis $\mathrm{C}$ virus infection. A prospective multicenter study of 321 patients. Medicine 2000;79:47-56.

2 Agnello V, De Rosa FG. Extrahepatic manifestations of HCD infection: some current issues. J Hepatol 2004;40:341-52.

3 Flint M, McKeating JA. The role of the Hepatic C virus glycoproteins in infection. Rev Med Virol 2000;10:101-17.

4 Khattab MA, Elsam M, Alavian SM. Hepatitis C virus as a multifaceted disease: a simple and updated approach for extrahepatic manifestations of hepatitis $\mathrm{C}$ virus infection. Review article. Hepatol Mon 2010;10:258-69.

5 Pascual M, Perrin L, Giostra E, et al. Hepatitis C virus in patients with cryoglobulinemia type II [letter]. J Infect Dis 1990;162:569-70.
6 Zignego AL, Giannini C, Ferri C. Hepatitis C virus-related lymphoproliferative disorders: an overview. World J Gastroenterol 2007;13:2467-78.

7 Lunel F, Cacoub P. Treatment of autoimmune and extrahepatic manifestations of hepatitis C virus infection. J Hepatol 1999;31 (Suppl 1):210-16.

8 Pubmed Health. Cryogobulinaemia. Created January 242011. http://www.ncbi.nlm.nih.gov/pubmedhealth/PMH0001567 (last accessed 24 Oct 2012)

9 Tarantino A, Campise M, Banfi G, et al. Long term predictors of survival in essential mixed cryoglobulinemic glomerulonephritis. Kidney Int 1995;47:618-23.

10 Lidove O, Cacoub P, Maisonobe T, et al. Cryoglobulinaemia and hepatitis $\mathrm{C}$ : worsening of peripheral neuropathy after interferon treatment. Gastroenterol Clin Biol 1999;23:403-6.

11 Ferri C, Zignego AL, Pileri SA. Cryoglobulins. J clin Pathol 2002;55:4-13.

12 Iannuzzella F, Vaglio A, Garini G. Management of hepatitis C virus-related mixed cryoglobulinemia. Am J Med 2010; 123:400-8.

13 Zaja F, De Vita S, Mazzaro C, et al. Efficacy and safety of rituximab in type II mixed cryoglobulinaemi vasculitis. Curr Opin Rheumatol 2008;20:23-8.

14 Pietrogarande M, De Vita S, Zignego AL, et al. Recommendations for the management of mixed cryoglobulinaemia syndrome in hepatitis C virus-infected patients. Autoimmun Rev 2011;10:444-54.

15 Kamar N, Rostaig L, Alric L. Treatment of hepatitis C-virus-related glomerulonephritis. Mini Review. Kidney Int 2006;69:436-9.

16 Chadban SJ, Atkins RC. Glomerulonephritis. Lancet 2005;365:1797-806.

17 Sabry AA, Sobh MA, Sheaashaa MA, et al. Effect of combination therapy (ribivarin and interferon) in HCV-related glomuerulonephropathy. Nephrol Dial Transplant 2002;17:1924-30.

18 Sansonno D, De Re V, Lauletta G, et al. Monocloonal antibody treatment of mixed cryoglobulinaema resistant to interferon alpha with an anti CD-20. Blood 2003;101:3818-26.

19 Phillips JD, Bergonia HA, Reilly CA, et al. A porphomethene inhibitor of uroporphyrinogen decarboxylase causes porphyria cutanea tarda. Proc Natl Acad Sci USA 2007;104:5079-84.

20 Gisbert JP, Garcia-Buey L, Pajares JM, et al. Prevalence of hepatitis $\mathrm{C}$ virus infection in porphyria cutanea tarda: systematic review and meta-analysis. J Hepatol 2003;39:620-7.

21 Fernandez I, Castellano G, de Salamanca RE, et al. Porphyria cutanea tarda as a predictor of poor response to interferon alfa therapy in chronic hepatitis C. Scand J Gastroenterol 2003;38:314-19.

22 Ryan Caballes Sendi H, Bonkovsky HL, et al. Hepatitis C, porphyria cutanea tarda and liver iron: an update. Liver Int 2012;32:880-93.

23 Frank J, Poblete-Gutierrez P. Porphyria cutanea tarda-when skin meets liver. Best Pract Res Clin Gastroenterol 2010;24:735-45. 